\title{
A MULTIPLE MANUAL REGISTER FOR DIFFERENTIAL LEUCOCYTE COUNTS
}

BY

\author{
C. SANDERS AND A. E. BENNETT \\ From the Medical and Electronics Divisions, Atomic Energy Research Establishment, \\ Harwell
}

(RECEIVED FOR PUBLICATION JANUARY 13, 1950)

One of the most trying and time-consuming operations in routine haematology is that of the differential leucocyte count. The method used in common practice is to write down on paper the different types of leucocytes as they are seen. To do so it is necessary for the person performing the count to be frequently looking away from his microscope. With a binocular microscope this routine can become very tiring, especially where many counts have to be made. With the monocular microscope also a great deal of time is lost. The multiple manual register was designed to overcome these difficulties. Experience with this tabulator has proved how valuable an asset it can be in a laboratory where many differential leucocyte counts have to be made, the time saved is considerable, and the general procedure of doing differential leucocyte counts has been eased to a most marked degree.

This instrument, which was produced by the Atomic Energy Research Establishment for the haematology department of the medical division, has been in use for several months. Because of the possible significance of the Arneth counts in cases of radiation exposure, the manual register was designed so as to allow for the differential leucocyte count and the Arneth count to be performed at the same time.

The instrument is designed to be operated by the left hand in order to leave the right hand free to operate the mechanical stage and fine focusing adjustment of the microscope. On this basis the lay-out of the different keys of the register has been arranged so as to place the keys of the leucocytes most commonly seen in the most convenient position for the operator. An even greater saving of time is effected if an assistant operates the register for the person doing the differential counts: It is a great help to the haematologist in studying the cytology of the blood film to be free of the necessity of interrupting his observations.

The multiple manual register differs from the "marbel blood cell calculator" which has been on the market for some time, in that the manual register possesses 13 different tabulator keys, compared with the eight keys numbered numerically, of the " marbel calculator"; furthermore the keys on the manual register are lettered for the different types of leucocytes commonly seen, and also with five numerals, 1 to 5 , for the Arneth count (Fig. 1). These keys are easily detached by removing a small screw, and, as suggested by other workers, could be replaced with suitably indexed keys for bone marrow cell counts. The indicator lettering above each indi- 
vidual register, corresponding to that on the appropriate key, may also be simply exchanged for any other form, representing a new set of indexed tabulator keys. The multiple manual register could be adopted for many purposes other than medical.

\section{Mechanical Details of the Register}

The multiple manual register (Fig. 1) incorporates 13 independent numerical registers. Each of these registers comprise two counting wheels (a units and a tens wheel) linked

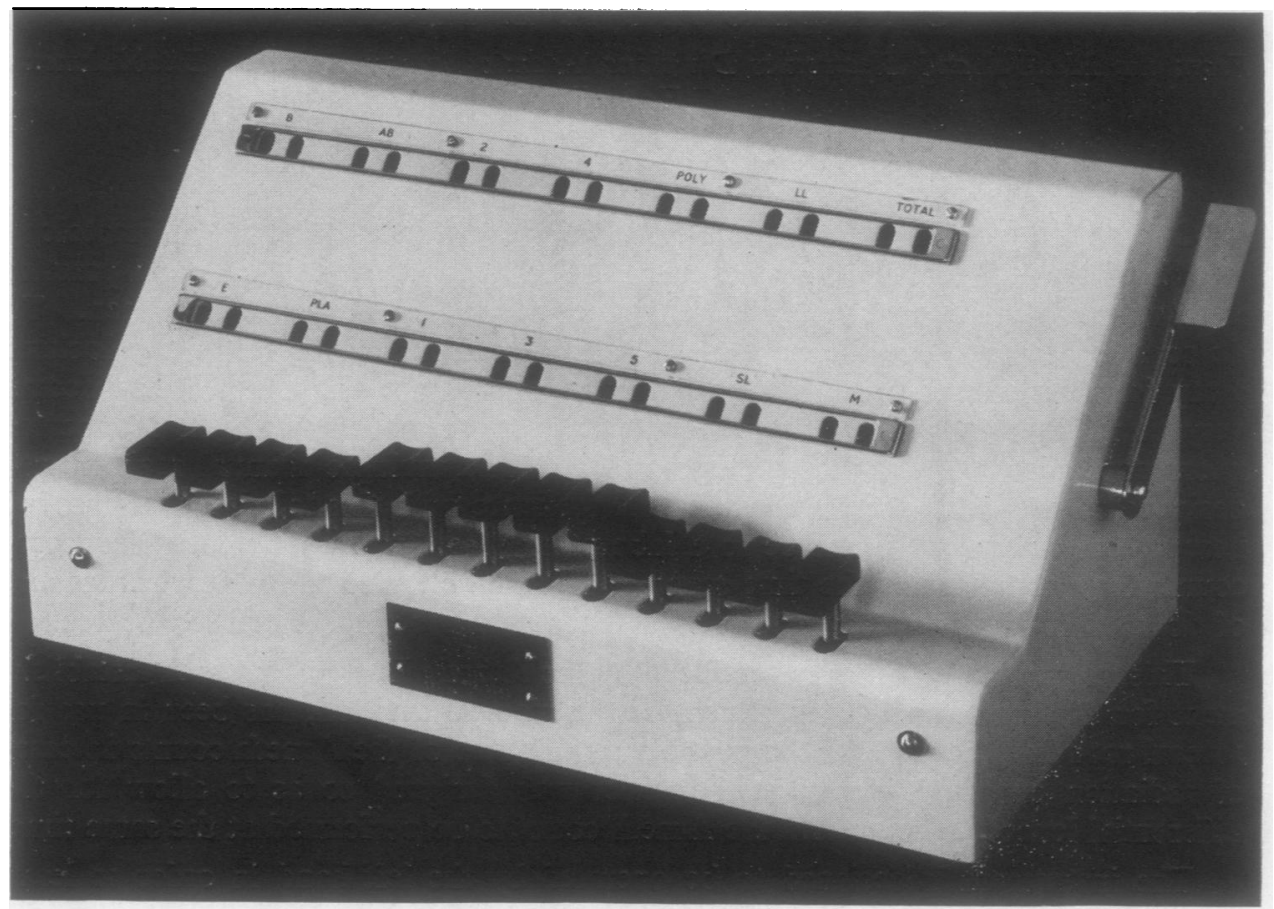

FIG. 1. - The register described.

$\mathrm{E}=$ Eosinophil. $\quad \mathbf{B}=$ Basophil. $\quad$ PLA $=$ Plasma Cell. $\quad$ AB $=$ Abnormal Cell. $1,2,3,4,5=$ Arneth, 1, 2, 3, 4, 5 .

POLY $=$ Polymorph. $\quad$ SL $=$ Small Lymphocyte. $\quad$ LL $=$ Large Lymphocyte. $\quad M=$ Monocyte.

together by a transfer pinion. This allows each register to record up to a maximum of 100 cells. The units drum of each register is coupled to a ten-toothed ratchet wheel, which is operated by a link mechanism from a key on the front of the instrument. The 13 keys are arranged in three groups of 4,5 , and 4 respectively, and are engraved (see key to Fig. 1) to represent eosinophil, basophil, plasma cell, abnormal cell (Arneth $1,2,3,4$, and 5), polymorph, small lymphocyte, large lymphocyte, and monocyte respectively. In addition to these registers there is a totalizing register operated by a bar linked to all 13 of the independent registers, which records a count each time any one of the 13 keys is pressed. It has two counting wheels and can therefore only record up to a total of 100 . It is, however, coupled to a small bell in such a way that this bell is struck as the register changes from $99-00-i . e$. , every time a count of 100 is registered. 
The complete set of registers may be reset to zero by the operation of a single lever mounted on the right-hand side of the instrument. To make the unit compact the various registers are assembled on two main spindles which are mounted so as to give a sloping faced unit which is easily read. The overall size of the register is 13 in. $\times 6 \frac{1}{2}$ in. $\times 6$ in.

An improved model is being constructed which will allow the total Arneth count to be recorded independently of the overall total. This will be achieved by a second bar mechanism being fitted which will only be operated when any one of the five Arneth register keys are pressed. (These keys will still operate the main bar which covers all the 13 independent registers.) This subsidiary bar mechanism will be coupled to a second totalizing register consisting of two counting wheels-i.e., it will record up to 100 cells. It will also be fitted with a bell mechanism so that an alarm is given as this (the Arneth total) register changes from 99-00.

This new model will also have three instead of two counting wheels in the main totalizing register-i.e., it will record up to 1,000 cells. However, its alarm bell will be struck every hundredth count.

The reset action will be the same: one lever will reset to zero all the registers in the instrument.

\section{Summary}

A multiple manual register for differential leucocyte counts is described. The register incorporates 13 independent keys arranged in three groups of 4, 5, and 4 respectively, the middle five keys numerically lettered for the Arneth count. A bell signals each time that a total of 100 cells is registered. The adaptability of this register for other purposes than the differential leucocyte count has been suggested.

Acknowledgment is made to the Director, Sir John Cockcroft, Atomic Energy Research Establishment, Harwell, for his permission to publish this article, also to Dr. K. Williams, P.M.O., for help given in the preparation of this paper.

English Numbering Machines, Ltd., have a development contract to engineer the improved model. The probable price is about $£ 15$. Owing to restrictions in dollar expenditure the "marbel blood cell calculator" is now unobtainable in this country. 